Mathématiques et sciences humaines
Mathematics and social sciences

192 | Hiver 2010

Varia

\title{
Evolution des nages et théorie des jeux
}

Game theory and the evolution of swimming

\section{Luc Collard}

\section{(2) OpenEdition}

\section{Journals}

Édition électronique

URL : http://journals.openedition.org/msh/11912

DOI : $10.4000 / \mathrm{msh} .11912$

ISSN : 1950-6821

\section{Éditeur}

Centre d'analyse et de mathématique sociales de l'EHESS

\section{Édition imprimée}

Date de publication : 15 décembre 2010

Pagination : 41-55

ISBN : 09876936

ISSN : 0987-6936

\section{Référence électronique}

Luc Collard, «Evolution des nages et théorie des jeux », Mathématiques et sciences humaines [En ligne], 192 | Hiver 2010, mis en ligne le 31 mars 2011, consulté le 23 juillet 2020. URL : http:// journals.openedition.org/msh/11912; DOI : https://doi.org/10.4000/msh.11912 


\title{
ÉVOLUTION DES NAGES ET THÉORIE DES JEUX
}

\author{
Luc COLLARD 1
}

\begin{abstract}
RÉSUMÉ - Nous nous servons de la Théorie des jeux pour formaliser une situation problématique observée en natation sportive lors de Championnats d'Europe espacés de trente ans. Dans les épreuves de $100 \mathrm{~m}$ de Dos, Papillon et Nage libre, les nageurs de haut niveau nagent plus vite en immersion qu'en émersion. En petits bassins $(25 \mathrm{~m})$, la règle des $15 \mathrm{~m}$ permet aux nageurs de rester sous l'eau sur des distances supérieures aux distances nagées en surface. Pourtant, les experts n'utilisent pas cette option et nagent plus en surface qu'en immersion. La modélisation de ce dilemme opposant tactique de surface (Surface+) à tactique d'immersion (Coulée+) permet de pronostiquer l'évolution des techniques de nage. Six cas de figures se profilent, résolus par l'équilibre de Nash, le Maximin et la Stratégie Évolutionnairement Stable (Evolutionary Stable Strategy). Dans les épreuves courtes, la technique sous-marine du dauphin - la cinquième nage - est l'intrus amené à prendre la place du réplicateur dominant, malgré les efforts de l'institution sportive qui dresse artificiellement les nageurs à garder la surface.
\end{abstract}

MOTS-CLÉS - Cinquième nage, Natation sportive, Stratégie Évolutionnairement Stable, Théorie des jeux

SUMMARY - Game theory and the evolution of swimming strokes

We used game theory to formalize a situation observed in competitive swimming, with a focus on European Championships thirty years apart. High-level swimmers move faster underwater than at the surface. In short-course $(25 \mathrm{~m}$ pool) events, the 15-metre rule authorizes swimmers to swim further underwater than they do at the surface. However, competitive swimmers in $100 \mathrm{~m}$ backstroke, butterfly and freestyle events in short-course pools do not take advantage of this option and spend more time at the surface than underwater. By modelling a dilemma opposing a surface tactic (Surface+) and an underwater tactic (Underwater+), it was possible to predict the evolution of swimming techniques. Six situations appeared and were resolved by Nash equilibrium, a Maximin strategy and an Evolutionary Stable Strategy. In short-course events, underwater dolphin kicking (the "fifth stroke") is a new "intruder" and is likely to become the dominant replicator - despite the efforts from swimming institutions, which artificially trains swimmers to stay at the surface.

KEYWORDS - Fifth stroke, Evolutionary Stable Strategy, Swimming sport, Theory of games

\section{1. "BERKOFF BLAST-OFF” (LE DÉCOLLAGE DE BERKOFF)}

Le 23 janvier 2008, l'ancien médaillé olympique David Berkoff est interrogé pour www.swimnetwork.com sur ce qui, vingt-quatre ans auparavant, l'a amené à nager sous l'eau en compétition comme un dauphin :

\footnotetext{
${ }^{1}$ Équipe d'accueil Information, Organisation et Action (EA 4260), UFR STAPS de Caen, Université de Caen Basse-Normandie (UCBN), boulevard du Maréchal Juin, Campus II, 14032 Caen cedex, luc.collard@orange.fr
} 
J'ai fait $15 \mathrm{~m}$ en ondulations après le départ pour faire le malin et, en regardant derrière moi, j'ai vu que je les avais tous grillés.

Alors étudiant en anthropologie, Berkoff oublie de préciser qu'il était membre de l'association Delphys organisatrice d'expériences aquatiques avec les dauphins [Van Eersel, 1993]. Entre 1984 et 1988, les coulées de ce nageur américain passeront de 15 à $30 \mathrm{~m}$; parallèlement, ses prestations sur $100 \mathrm{~m}$ Dos décolleront.

Et qu'est-ce que ça fait d'être le nageur ayant poussé la Fédération [FINA : Fédération Internationale des Nageurs Amateurs] à limiter les coulées à $15 \mathrm{~m}$ après le plongeon et chaque virage? lance le journaliste, David Cromwell.

(La règle s'est d'abord appliquée au Dos [1988] puis au Papillon [1996] et enfin à la Nage libre [2001] - la Brasse n'étant pas concernée par cette limitation compte tenu d'autres contraintes obligeant les nageurs à faire rapidement surface).

La raison pour m'empêcher de poursuivre mes coulées était la "sécurité", explique Berkoff, parce qu'ils [les officiels de la FINA] ne voulaient pas que des gens se blessent en essayant d'en faire autant. Je leur ai fait remarquer que ça ne les dérangeait pas que les nageuses de Natation Synchronisée réalisent fréquemment des apnées dynamiques de plus d'une minute.

Les records masculins sur $100 \mathrm{~m}$ dans les quatre nages légales n'excèdent pas la minute. Sur $50 \mathrm{~m}$ déjà, de nombreux Crawleurs et Papillonneurs maintiennent l'apnée sur la totalité de la distance. En bassin olympique $(50 \mathrm{~m})$ ou en petit bassin $(25 \mathrm{~m})$, il y a respectivement une et trois possibilités de respirer avant chaque virage en nageant dans la technique du dauphin, soit environ toutes les 30 ou 15 secondes. On voit bien que l'argument respiratoire ne tient pas. Une cinquième nage est du domaine du possible. Alors pourquoi l'avoir muselée ? Lorsqu'une nouvelle technique plus performante émerge dans un sport où seul le chronomètre compte, n'est-il pas logique de lui laisser une place ? Peut-on condamner des nageurs pour excès de vitesse?

Les détracteurs du "Berkoff blast-off" («Berkoff décolle » : surnom donné par les médias à ce nageur dans les années 80 , tant l'avance prise au moment de ses coulées au départ était conséquente) font justement remarquer que son record du monde a été maintes fois battu depuis 1988 par des Dossistes tous limités à $15 \mathrm{~m}$ de coulées - y compris par lui-même, aux Jeux de Barcelone [1992]. Berkoff ne nie pas les progrès de la natation sportive à mesure qu'elle a refréné les possibilités de s'immerger. Mais il ne peut s'empêcher de revenir sur le potentiel de la nage dauphin par l'évocation d'une nouvelle individualité : Ryan Lochte. Durant l'été 2009, Lochte sera effectivement chronométré à l'entraînement en 20,8 secondes sous l'eau sur $50 \mathrm{~m}$, avec une seule inspiration avant le virage du $25 \mathrm{~m}$. C'est la meilleure performance de tous les temps sur cette distance, toutes nages confondues, départ dans l'eau.

Au-delà de l'anecdote, l'observation de l'évolution des performances et du pourcentage des coulées par bassin dans des épreuves officielles depuis une trentaine d'années devrait fournir les informations nécessaires pour poser le dilemme : avouer la suprématie des coulées tout en niant la possibilité qu'elles puissent prendre le dessus sur les nages de surface.

Le Tableau 1 ci-dessous présente l'évolution des performances du $100 \mathrm{~m}$ dans les 4 nages sur trois compétitions internationales masculines. À l'époque des championnats d'Europe en petit bassin $(25 \mathrm{~m})$ de 1982, il n'y a pas de limite de distance avant que la tête des nageurs ne coupe la surface. En 2002 comme en 2010, il n'est plus permis aux nageurs de sortir au-delà de la ligne des $15 \mathrm{~m}$ en Dos, en Nage libre (épreuve systématiquement nagée en Crawl) et en Papillon. Mais « $15 \mathrm{~m}$ » représentent près des deux tiers d'un bassin de $25 \mathrm{~m}$. On peut ainsi voir si les nageurs passent plus de temps immergés qu'en surface. 


\begin{tabular}{lcccccc}
\hline $\begin{array}{c}\text { Épreuves en petit basin } \\
(25 \mathrm{~m}) / \text { Prestations des } \\
\text { vainqueurs des } \\
1 / 2 \text { finales hommes }\end{array}$ & Année & $\begin{array}{c}\text { Temps } \\
\text { moyen } \\
(\mathrm{s})\end{array}$ & $\begin{array}{c}\text { Coulée après le } \\
\text { plongeon }(\mathrm{m})^{*}\end{array}$ & $\begin{array}{c}\text { Coulée après } \\
\text { les virages } \\
(\mathrm{m})^{*}\end{array}$ & $\begin{array}{c}\text { Pourcentage de } \\
\text { distance nagée en } \\
\text { dauphin }\end{array}$ & $\begin{array}{c}\text { Nombre moyen de } \\
\text { coups de bras en } \\
\text { surface }\end{array}$ \\
\hline \multirow{2}{*}{100 m Papillon } & 1982 & 54,1 & 7 & 5 & $22 \%$ & 40,0 \\
& 2002 & 51,4 & 13 & 11 & $46 \%$ & 28,5 \\
& 2010 & 51,3 & 13 & 11 & $46 \%$ & 28,0 \\
\hline \multirow{3}{*}{100 m Dos } & 1982 & 56,3 & 7 & 4 & $19 \%$ & 60,5 \\
& 2002 & 52,2 & 15 & 10 & $45 \%$ & 51,0 \\
100 m Brasse & 2010 & 50,3 & 15 & 13 & $54 \%$ & 42,0 \\
\hline \multirow{3}{*}{100 m Nage libre } & 1982 & 62,2 & 7 & 6 & $25 \%$ & 45,5 \\
& 2002 & 59,7 & 11 & 9 & $38 \%$ & 38,0 \\
& 2010 & 58,3 & 13 & 10 & $43 \%$ & 34,5 \\
\hline
\end{tabular}

\footnotetext{
* arrondi au nombre entier le plus proche
}

TABLEAU 1. Comparaison de plusieurs indicateurs lors des $1 / 2$ finales des épreuves de $100 \mathrm{~m}$ messieurs de Championnats d'Europe en bassin de $25 \mathrm{~m}$

Moins les nageurs passent de temps en surface, plus ils sont rapides. Pour autant les nageurs passent plus de temps en surface qu'en immersion. En 1982, la distance des coulées n'est pas réglementée ; en 2002-10 elle est limitée à $15 \mathrm{~m}$ après chaque poussée du mur, soit $60 \mathrm{~m}$ sur 100 .

Cela ne surprendra personne. On nage aujourd'hui plus vite qu'il y a vingt ans. Plus surprenant est d'observer les nageurs « de l'ancien temps » se précipiter en surface juste après les virages alors que rien ne les y obligeait. On était tellement persuadé à l'époque d'être moins efficace sous l'eau ! En vingt ans, les coulées ont grosso modo doublé en distance. Par voie de conséquence, le nombre moyen de coups de bras par bassin a chuté. Ces différences sont hautement significatives entre 1982 et $2002\left(\mathrm{Khi}^{2}\right.$, $p<0.01)$. À l'intérieur de chaque nage, la corrélation de rang est de $K=+1$ entre les performances chronométriques et les pourcentages de distances nagées en dauphin.

Cependant, l'observation attentive des résultats ne peut donner complètement raison au "Berkoff blast-off". Dans les quatre nages, en 2002 et 2010, les prestations immergées n'atteignent jamais les $60 \%$ autorisées $(15 \mathrm{~m} \times 4=60 \mathrm{~m})$. Elles sont même malgré le plongeon de départ, et à une exception près, en deçà de $50 \%$. L'inventeur de la cinquième nage n'a peut-être pas tort lorsqu'il dit que nager sous l'eau va plus vite que nager en surface. Mais à condition que cela soit sur des distances inférieures aux distances nagées en surface !

Finalement, les nageurs se soumettent à une double contrainte :

CONSTAT 1. «Dans les épreuves de $100 \mathrm{~m}$ en bassin de $25 \mathrm{~m}$, en Papillon, Dos et Nage libre, les nageurs de haut niveau nagent plus en surface qu'en immersion alors qu'ils n'y sont pas obligés » (Surface+). Ils doivent avoir de bonnes raisons pour cela : rendement (surtout en Crawl), gestion de l'effort, coordination de la respiration, facilité d'observation des concurrents, habitudes de compétitions et d'entraînements, etc. Ce constat s'appuie sur l'observation de la majorité (Tableau 1, avant dernière colonne).

Quelques rares nageurs - parmi les meilleurs - ne s'y soumettent pas. Outre Berkoff, le Russe Pankratov fut probablement le trublion déclencheur de la limitation 
des coulées en Papillon. En 1995, lors d'une épreuve de 100 m Papillon, au cours d'une étape du championnat du monde en petit bassin $(25 \mathrm{~m})$, il scotcha les organisateurs en traversant en dauphin l'intégralité du premier bassin, profondément immergé, n'émergeant qu'à l'orée du mur pour effectuer un unique mouvement de papillon. Son temps au toucher : 10,5 secondes, digne des meilleurs chronos actuels sur $25 \mathrm{~m}$ Crawl. A cette période, l'Américaine Hyman bat également le record du monde du $100 \mathrm{~m}$ Papillon avec $35 \mathrm{~m}$ de coulée, dans un style que les spécialistes nommeront: "Fish kick" (ondulations du corps dans un plan vertical, juste sous la surface, à l'instar des poissons). Plus récemment, l'Allemand Rupprath ou le Russe Vyatchanin nagent plus de $50 \%$ des épreuves de $100 \mathrm{~m}$ et $200 \mathrm{~m}$ Dos et Papillon en immersion [Collard, 2009]. Le Français Leveaux doit son passage sous la barre des 45 secondes au 100m Nage libre à ses qualités d'ondulation. En 2008, il est le premier crawleur à effectuer trois de ses quatre coulées du $100 \mathrm{~m}$ au-delà des $10 \mathrm{~m}$. Au total, il passe $45 \%$ de sa course totalement immergé et ne réalise que 22 cycles de bras en surface (au lieu des 30 habituels, 30 cycles $=60$ coups de bras).

L'observation de cette minorité triomphante, associée aux comparaisons d'évolution des performances et des distances d'immersion sur vingt ans (Tableau 1, $3^{\mathrm{e}}$, $4^{\mathrm{e}}$ et $5^{\mathrm{e}}$ colonne) amène à la rédaction d'un second constat.

CONSTAT 2. «Dans les épreuves de $100 \mathrm{~m}$ en bassin de $25 \mathrm{~m}$, les nageurs les plus performants sont ceux qui exécutent les coulées les plus longues »(Coulée+).

L'énoncé de ces deux régularités et leur formalisation à l'aide de la Théorie des jeux permettront-ils de régler le différend opposant :

- Ceux qui ne jurent que par les déplacements en surface : la majorité des nageurs experts restent émergés sur des distances supérieures à ce qu'impose le règlement. L'institution sportive, ses représentants (les entraîneurs) et de nombreux biomécaniciens de la natation militent activement en faveur de l'exclusivité de ces techniques de nage : Dos, Brasse, Papillon, Crawl [Holt, 1989 ; Sprigings, Koehler, 1990 ; Sanders, 1997 ; Catteau, 2008] et :

- Ceux qui n'ont d'yeux que pour les immersions : les nageurs dissidents s'illustrent par leur supériorité dans cette cinquième nage [Counsilman, 1968, Schleihauf, 1978, Maglischo, 2003, Collard, 2007] ; d'ailleurs, la natation sportive de surface va à contresens de l'adaptation des mammifères au milieu aquatique [Fish, 1993, Thewissen, Hussain, Arif, 1994, Spoor, Bajpai, Hussain, Kumar, Thewissen, 2002, Thewissen, Cooper, Clementz, Bajpai, Tiwari, 2007]. Hertel [1966] estime que nager en surface peut augmenter les résistances à l'avancement par cinq - c'est sans doute la raison pour laquelle aucun des mammifères marins ayant reconquis le milieu marin depuis 55 millions d'années n'utilise ce mode de propulsion.

La Théorie des jeux a principalement pour objet de prédire les comportements de joueurs compte tenu des règles d'un jeu - jeu qui peut être créé de toutes pièces à partir des traits caractéristiques de la situation. À ce titre, il est logique que le sport et les jeux sportifs fassent partie de ses champs d'application. Pourtant, comme l'annonce à regret Pierre Parlebas [2005]:

À côté des mathématiques utiles, les jeux apparaissaient bien futiles [p. 5].

Car c'est bien là que gît le lièvre, poursuit-il : la mathématisation des jeux apporte-t-elle du nouveau? Permet-elle d'accroître notre savoir et notre pouvoir d'intervention sur le terrain? Autrement dit, cet outillage technique favorise-t-il un enrichissement de nos connaissances relatives au jeu sportif? [p. 6]. 
Tel est le pari de cet article : formaliser au plus juste le conflit opposant ces deux tactiques de nage pour voir comment la natation sportive va évoluer dans ses techniques. La Section 2 proposera une formalisation du dilemme Surface+ versus Coulée + qui s'apparente au conflit bien connu entre intérêts individuels (Coulée+) et choix collectif (Surface+). Plusieurs modèles de jeu (La parabole des chasseurs, La fureur de vivre, Le dilemme du prisonnier) viendront tour à tour expliquer le dilemme des nageurs. La Section 3 illustrera l'adaptation dans le temps des différents modèles. Pour cela, nous ferons une incursion dans le domaine de la Théorie des jeux évolutionnaire. Nous serons notamment aidé en cela par un spécialiste de la mécanique des fluides, Maynard Smith [1958] - qui aurait très bien pu s'intéresser aux techniques de nages - mais qui s'est finalement converti à la Théorie de l'évolution en lui appliquant avec succès la Théorie des jeux.

\section{DEUX TACTIQUES, 4 STRATÉGIES, 6 POSSIBILITÉS}

Imaginons la confrontation de champions de natation ayant exactement les mêmes qualités de nage (on évite ainsi de devoir tenir compte du niveau d'expertise : niveau qui tend d'ailleurs à s'homogénéiser, tant les techniques d'entraînement et de sélection sont uniformes). Le jeu est à 8 joueurs et à somme non nulle, chacun occupant une ligne d'eau de 2,5 $\mathrm{m}$ de large dans le bassin de $25 \mathrm{~m}$ de long. Le jeu est répété (la répétition correspond à la redondance des compétitions).

Les nageurs ont le choix entre Surface+, c'est-à-dire nager plus en surface qu'en immersion ; et Coulée +, c'est-à-dire le contraire. Il vient une matrice à double entrée et à 4 stratégies pures (Tableau 2). En ligne, on place le joueur nageur (au singulier), en colonne les $\mathrm{N}-\mathrm{l}$ autres nageurs que, pour simplifier, nous considérerons comme un super-joueur : Nageurs (avec une majuscule et au pluriel).

\begin{tabular}{|c|c|c|c|}
\hline \multirow{2}{*}{\multicolumn{2}{|c|}{$\pi$}} & \multicolumn{2}{|c|}{ Nageurs } \\
\hline & & Coulée+ & Surface+ \\
\hline \multirow{2}{*}{ nageur } & Coulée+ & $(\mathrm{A}, \mathrm{A})$ & $(\mathrm{C}, \mathrm{D})$ \\
\hline & Surface+ & $(\mathrm{D}, \mathrm{C})$ & $(\mathrm{B}, \mathrm{B})$ \\
\hline
\end{tabular}

TABLEAU 2. Matrice de satisfaction des nageurs

Les lettres entre parenthèses correspondent au niveau de satisfaction. La première lettre dans la parenthèse correspond au gain de nageur; la seconde est pour Nageurs.

Nous nous servons des CONSTATS issus des données du Tableau 1 pour rendre compte des degrés de satisfaction. Entendons-nous sur «satisfaction ». Il ne s'agit pas du niveau subjectif de plaisir éprouvé par les nageurs, ni du désir plus ou moins avoué des entraîneurs (même si ces désirs sont une cause indirecte probable de ce que nous allons désigner comme relevant du degré de satisfaction). Il ne s'agit pas non plus du niveau absolu de performance - qui divise aujourd'hui encore, comme je l'ai écrit plus haut, scientifiques et représentants de l'institution sportive : compte tenu de leurs prédispositions anthropométriques et des grands principes de la mécanique des fluides, les nageurs sont-ils capables d'entretenir sous l'eau une vitesse supérieure à celle obtenue en surface ? Par "satisfaction », nous désignons le niveau d'efficacité relatif d'une tactique ou d'une stratégie sur une autre lors des prestations aquatiques observées. Depuis trente ans dans les épreuves de $100 \mathrm{~m}$ (Tableau 1), la quasi totalité des nageurs experts passent plus de temps en surface qu'en immersion, alors qu'ils n'y sont pas 
obligés (CONSTAT 1). Comme ils n'ont tous qu'une idée en tête : battre les autres, nous en déduisons qu'il est plus satisfaisant pour eux de faire ainsi. Cela nous permet d'affirmer que la combinaison (Surface+, Surface +$)=(\mathrm{B}, \mathrm{B})$ domine la stratégie $($ Coulée + , Coulée +$)=(\mathrm{A}, \mathrm{A})$, d'où : $\mathrm{B}>\mathrm{A}$. Dit autrement, nous aurions annoncé $\mathrm{A}>\mathrm{B}$, si les $60 \%$ d'immersions autorisées par bassin étaient approchés. On en est aujourd'hui à $34 \%$, dans la nage la plus rapide (Nage libre, dernière ligne du Tableau 1)!

Mais le paradoxe tient à ce que dans le même temps (CONSTAT 2), les nageurs les plus rapides s'illustrent lors de coulées plus longues réalisées face à des adversaires retrouvant prématurément la surface : $\mathrm{C}>\mathrm{D}$ (Tableau 2). Sur le plan diachronique, le gain de temps est corrélé positivement avec la distance des parties nagées sous l'eau (Tableau 1). Sur le plan synchronique, de deux ou plusieurs nageurs immergés (de niveau comparable, tel que supposé et y compris en épreuve de Nage libre), c'est plus souvent le dernier à couper la surface qui l'emporte. Dans le jargon de la natation on dit « qu'il passe sous la vague des autres ». La stratégie aboutissant à $(C, D)$ va au profit de celui des deux qui joue Coulée +. Mais alors pourquoi tous les nageurs n'endossent-ils pas cette tactique ? C'est bien l'énigme que nous tentons de percer. En effet, il se pourrait que le profit (C) obtenu par nageur jouant Coulée+ contre Nageurs jouant Surface+ (D) s'estompe face à Nageurs jouant lui-même Coulée+ (A, A) - il n'y a plus de passage sous la vague qui vaille, chacun pouvant même subir les turbulences du vortex générées par les ondulations des autres - au point que nageur ait finalement plus à gagner à rester en Surface+ si Nageurs fait de même $(\mathrm{B}, \mathrm{B})$... Ainsi se pose le problème du niveau hiérarchique de satisfaction entre $\mathrm{A}$ et $\mathrm{C}, \mathrm{A}$ et $\mathrm{D}, \mathrm{B}$ et $\mathrm{C}, \mathrm{B}$ et $\mathrm{D}$.

La Figure 1 présente le graphe des rapports de domination compte tenu des CONSTATs 1 et 2. On ignore l'orientation de 4 des 6 arcs. Pour résoudre le jeu, il faut envisager les 6 cas de figures possibles. En attribuant arbitrairement un point par majorité, on établit une matrice des gains pour chacune des six possibilités (Tableau 3). Choisir un pas uniforme de +1 point par majorité (on aurait pu aussi bien choisir $+n$, une constante positive quelconque) n'est pas indifférent. Pour un tournoi transitif comme $\mathrm{B}>\mathrm{A}>\mathrm{C}>\mathrm{D}$ par exemple, si l'écart de points entre $\mathrm{B}$ et $\mathrm{A}$ n'était pas proportionnel à l'écart entre $\mathrm{A}$ et $\mathrm{C}$ (ou $\mathrm{C}$ et $\mathrm{D}$ ), il en résulterait une toute autre analyse. Mais nous ne disposons pas d'éléments issus de l'observation des nageurs permettant d'envisager de telles variations.

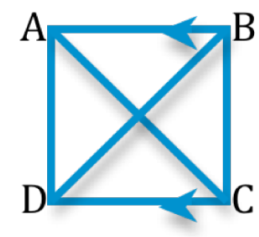

FIGURE 1. Graphe hiérarchique des sommets compte tenu des CONSTATS 1 et 2 Les CONSTATS 1 et 2 ne permettent d'orienter que 2 arcs sur 6 . 


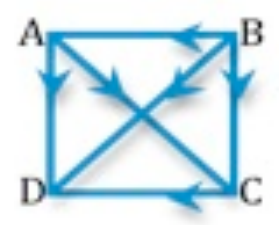

\begin{tabular}{|c|r|c|c|}
\hline \multirow{2}{*}{ CAS 1 } & \multicolumn{3}{|c|}{ Nageurs } \\
\hline \multirow{3}{*}{ nageur } & $\boldsymbol{\lambda}$ & Coulée+ & Surface+ \\
\cline { 2 - 4 } & Coulée+ & $(+2,+2)$ & $(+1,0)$ \\
\cline { 2 - 4 } & Surface+ & $(0,+1)$ & $(+3,+3)$ \\
\hline
\end{tabular}

CAS $1:$ B $>$ A $>$ C $>$ D

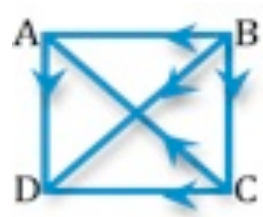

\begin{tabular}{|c|r|c|c|}
\hline \multirow{2}{*}{ CAS 2 } & \multicolumn{3}{|c|}{ Nageurs } \\
\hline \multirow{3}{*}{ nageur } & $\boldsymbol{\lambda}$ & Coulée + & Surface+ \\
\cline { 2 - 4 } & Coulée+ & $(+1,+1)$ & $(+2,0)$ \\
\cline { 2 - 4 } & Surface + & $(0,+2)$ & $(+3,+3)$ \\
\hline
\end{tabular}

CAS $2: \mathbf{B}>\mathbf{C}>\mathbf{A}>\mathbf{D}$

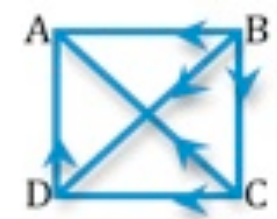

\begin{tabular}{|c|r|c|c|}
\hline \multirow{2}{*}{ CAS 3 } & \multicolumn{3}{|c|}{ Nageurs } \\
\hline \multirow{4}{*}{ nageur } & $\boldsymbol{\lambda}$ & Coulée + & Surface+ \\
\cline { 2 - 4 } & Coulée+ + & $(0,0)$ & $(+2,+1)$ \\
\cline { 2 - 4 } & Surface + & $(+1,+2)$ & $(+3,+3)$ \\
\hline
\end{tabular}

CAS $3: \mathbf{B}>\mathbf{C}>\mathbf{D}>\mathbf{A}$

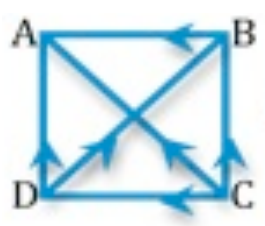

\begin{tabular}{|c|r|c|c|}
\hline \multirow{2}{*}{ CAS 4 } & \multicolumn{3}{|c|}{ Nageurs } \\
\hline \multirow{3}{*}{ nageur } & $\boldsymbol{\lambda}$ & Coulée+ & Surface+ \\
\cline { 2 - 4 } & Coulée+ + & $(0,0)$ & $(+3,+2)$ \\
\cline { 2 - 4 } & Surface+ & $(+2,+3)$ & $(+1,+1)$ \\
\hline
\end{tabular}

CAS $4:$ C $>$ D $>$ B $>$ A

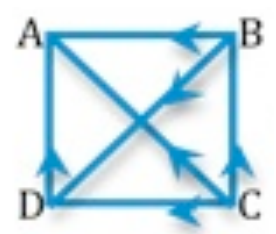

\begin{tabular}{|c|r|c|c|}
\hline \multirow{2}{*}{ CAS 5 } & \multicolumn{3}{|c|}{ Nageurs } \\
\hline \multirow{4}{*}{ nageur } & $\boldsymbol{\lambda}$ & Coulée+ & Surface+ \\
\cline { 2 - 4 } & Coulée+ + & $(0,0)$ & $(+3,+1)$ \\
\cline { 2 - 4 } & Surface+ + & $(+1+3)$ & $(+2,+2)$ \\
\hline
\end{tabular}

CAS $5:$ C $>$ B $>$ D $>$ A

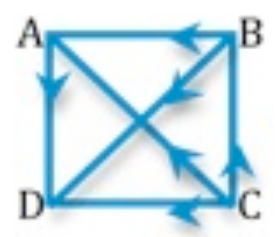

\begin{tabular}{|c|r|c|c|}
\hline \multirow{2}{*}{ CAS 6 } & \multicolumn{3}{|c|}{ Nageurs } \\
\hline \multirow{4}{*}{ nageur } & $\boldsymbol{\lambda}$ & Coulée + & Surface+ \\
\cline { 2 - 4 } & Coulée+ & $(+1,+1)$ & $(+3,0)$ \\
\cline { 2 - 4 } & Surface + & $(0,+3)$ & $(+2,+2)$ \\
\hline
\end{tabular}

CAS $6:$ C $>$ B $>$ A $>$ D

TABLEAU 3. Graphes de satisfactions et matrices de gains dans les 6 cas possibles Dans chaque graphe « >» signifie « plus satisfaisant que »; idem pour les matrices de gains : plus le chiffre est élevé, plus la satisfaction est grande. Le cas 3 est le seul donnant directement l'avantage à la tactique Surface+. Les autres nécessitent de repérer le moindre mal et l'équilibre de Nash. 


\section{CAS 1 ET 2. LA PARABOLE DES CHASSEURS}

Les cas $1(\mathrm{~B}>\mathrm{A}>\mathrm{C}>\mathrm{D})$ et $2(\mathrm{~B}>\mathrm{C}>\mathrm{A}>\mathrm{D})$ s'apparentent à La parabole des chasseurs de Rousseau [1756] dans son Discours sur l'origine de l'inégalité, repris par Raymond Boudon [1977] pour illustrer la notion «d'effet pervers » et par Pierre Parlebas [1986] pour expliquer la mécanique du contrat ludique. S'agit-il de prendre un cerf, explique Rousseau, chacun des chasseurs sait bien qu'il lui faut garder son poste : dans l'intérêt collectif et dans son propre intérêt particulier. Mais si un lièvre passe à portée de l'un d'eux, il y a grand risque que ce dernier le poursuive sans scrupule et, qu'ayant quitté son poste et atteint sa modeste proie, il se soucie fort peu de faire manquer le cerf au collectif. Telle est la situation interactionnelle au fondement du contrat social. La règle collective («il est interdit de chasser le lièvre pendant une chasse au cerf ») fait obstacle à l'effet pervers.

Dans les CAS 1 et 2 (Tableau 3), l'intérêt de tous est de garder la surface $(+3,+3)$. C'est un équilibre de Nash, optimum de surcroît. Aucun des nageurs n'a intérêt à changer de tactique, si l'autre maintient la sienne. Mais cette position n'est pas sans risque. En s'y prenant ainsi, chacun se met sous la dépendance de la bienveillance d'autrui. Si un nageur tourne casaque (comme le chasseur qui quitte son poste pour courser un lièvre), l'autre en Surface+ obtient la pire récompense : 0, et le contrevenant se satisfait d'un maigre +1 (CAS 1) ou +2 (CAS 2). Le niveau de sécurité, le Maximin (le Maximum du minimum de satisfaction au cas où le pire arriverait) est obtenu en jouant Coulée+: on garantit +1 , quoi que fasse l'autre. La stratégie (Coulée+, Coulée +) représente un second équilibre de Nash, sous-optimal celui-ci, mais plus sûr. Sans un Pouvoir central incitatif exerçant un droit de « sanction altruiste », les nageurs risquent de s'y coordonner.

\section{CAS 3. SANS ÉQUIVOQUE}

Le CAS 3 (B > C > D > A, Tableau 3) est le seul des 6 à être sans équivoque. Surface+ domine strictement Coulée+. Il n'y a aucun intérêt à rester immergé plus qu'on ne peut nager en surface. En s'écartant unilatéralement de la stratégie dominante, on passe de +3 à +2 , ce qui n'est pas trop pénalisant. Mais l'autre passe alors de +3 à +1 . Par représailles, ce dernier peut à son tour, au coup suivant, jouer Coulée+. Se coordonnant en (Coulée+, Coulée+), les nageurs obtiennent le pire score $(0,0)$. Aucune chance que cela se produise : la stratégie n'est ni équilibrée, ni sûre. Dans cette configuration, tout manquement à l'intérêt collectif (Surface+, Surface+) se soldera par une moindre satisfaction individuelle. L'institution n'est même pas obligée de punir les dissidents. Il n'y en aura pas.

\section{CAS 4 ET 5. POULE MOUILLÉE}

Dans les CAS $4(\mathrm{C}>\mathrm{D}>\mathrm{B}>\mathrm{A})$ et $5(\mathrm{C}>\mathrm{B}>\mathrm{D}>\mathrm{A})$, la solution nécessite le recours aux probabilités. La situation s'apparente cette fois-ci à La fureur de vivre, jeu inspiré du célèbre défi en voitures incarné à l'écran par James Dean [1955]. Le dernier qui saute de la voiture avant le ravin a gagné ; à moins que le perdant soit celui qui tombe dans le ravin, faute de ne pas avoir voulu s'éjecter le premier... On trouve une seconde appellation moins dramatique du jeu : «Poule mouillée ». D'un point de vue rationnel, le dilemme de Poule mouillée s'explique par l'absence de tactique dominante. Ni Coulée+, ni Surface+ ne domine dans l'absolu.

Jouer le maximin conduit a (surface + , surface + ) avec un gain garanti de $:(+1,+1)$ dans le CAS 4 , et $(+2,+2)$ dans le CAS 5 . Hélas, en jouant ce moindre mal, les nageurs se 
coordonnent dans une situation non équilibrée. Elle ne peut constituer la solution du jeu. Le CAS 5 (à l'instar du CAS 4) offre deux équilibres de Nash en stratégie pure : (coulée+, surface+) et (surface+, coulée+). Chaque nageur ayant intérêt a être celui des deux qui ondule, les joueurs risquent de se coordonner en (coulée+, coulée+) qui est la moins bonne des stratégies $(0,0)$.

Le jeu étant répété, il existe un autre équilibre de Nash mais en stratégie mixte (c'est-à-dire sur plusieurs parties, avec des probabilités de 0 à 1 ) permettant d'améliorer le sort des joueurs.

Soit $q$ la probabilité que Nageurs joue Coulée+ dans le CAS 5 :

Si nageur joue Coulée+, son espérance est $E=3(1-q)$;

Si nageur joue Surface+, son espérance est $E=q+2(1-q)$;

L'équilibre de Nash en stratégie mixte s'obtient avec $3-3 q=q+2-2 q$; d'où $q=1 / 2$ et $E q=+1,5$ point par course. La solution consistant à jouer une fois sur deux Coulée+ est la plus rationnelle. De même, si $r$ est la probabilité de nageur de Coulée+, on trouve $r=1 / 2$ et $E r=+1,5 \mathrm{Pt} / \mathrm{C}$ à l'équilibre. Il est facile de vérifier les mêmes résultats dans le CAS 4.

En examinant chaque possibilité selon les valeurs de $q$ et $r$, on trouve :

- Si $q<1 / 2$, nageur maximise ses gains (de $+1,5$ à +3 , CAS 4 et 5) en jouant avec $r=1$;

- Si $q=1 / 2$, nageur obtient $+1,5$ en jouant indifféremment Coulée + ou Surface+ ;

- Si $q>1 / 2$, nageur maximise ses gains (de $+1,5$ à +2 , cas 4 ; de +1 à $+1,5$, cas 5 ) en jouant avec $r=0$.

Cette situation de Poule mouillée représente ici le summum de l'ambiguïté : les nageurs devraient choisir alternativement Coulée+ et Surface+.

Notons que l'équilibre à $q=r=1 / 2$ s'obtient quels que soient les gains arbitraires affichés, pourvu qu'ils respectent la mécanique du jeu (Tableau 4).

CAS 4
\begin{tabular}{r|c|c|r|c|c|}
$\boldsymbol{\lambda}$ & Reste $^{*}$ & Saute** & $\boldsymbol{\lambda}$ & Reste & Saute \\
\hline Reste & $(\mathrm{U}-2),(\mathrm{U}-2)$ & $(\mathrm{U}+1),(\mathrm{U})$ & Reste & $(\mathrm{U}-1),(\mathrm{U}-1)$ & $(\mathrm{U}+2),(\mathrm{U})$ \\
\hline Saute & $(\mathrm{U}),(\mathrm{U}+1)$ & $(\mathrm{U}-1),(\mathrm{U}-1)$ & Saute & $(\mathrm{U}),(\mathrm{U}+2)$ & $(\mathrm{U}+1),(\mathrm{U}+1)$ \\
\hline
\end{tabular}

TABLEAU 4. Matrices de satisfaction de La fureur de vivre (ou Poule mouillée)

La matrice de gauche s'apparente au CAS 4 ; celle de droite au CAS 5. Reste correspond à

Coulée+ et Saute à Surface+. Le gain U peut prendre n'importe quelle valeur.

* Reste au volant de la voiture et continue à rouler vers le ravin malgré le danger ;

**Saute de la voiture pour ne pas tomber dans le ravin.

\section{CAS 6. LE DILEMME DU PRISONNIER}

Le CAS 6 s'apparente au Dilemme du prisonnier $(\mathrm{C}>\mathrm{B}>\mathrm{A}>\mathrm{D})$. Devenu un des modèles les plus célèbres de la théorie des jeux, c'est à Tucker [1950] que l'on doit son nom. Deux accusés sont détenus dans des cellules séparées. Leur choix se réduit à deux tactiques : avouer le crime, ou le nier. Si l'un des deux avoue et pas l'autre, le premier aura une récompense alors que le second sera lourdement condamné. Si aucun des deux n'avoue, faute de preuve ils seront libérés. Si les deux avouent, ils seront tous deux modérément condamnés. Avec une telle matrice des gains, l'intérêt collectif (Nie, Nie) 
peut être bafoué par la recherche individuelle de récompense. Anticipant cette trahison, les prisonniers risquent de se retrouver en (Avouer, Avouer).

À l'instar des prisonniers de Tucker, les nageurs en CAS 6 s'appuient sur la double contrainte de deux lois contradictoires.

Pour ce dilemme du nageur, nier représente le choix collectif optimum consistant à « nier » l'importance des coulées. Il correspond aux comportements de la majorité des nageurs énoncés en CONSTAT 1 : garder la surface (Surface+) lorsqu'il leur est encore permis de s'immerger. "Avouer », au contraire, correspond aux intérêts individuels énoncés en CONSTAT 2: s'immerger plus qu'on ne reste en surface (Coulée+); «avouer» la suprématie de la cinquième nage. La stratégie de l'aveu est le seul équilibre de Nash [1950, 1951]. On voit bien le dilemme : la situation de chacun est meilleure si aucun n'avoue (Surface+, Surface +$):(+2,+2)$, mais aucun des joueurs ne prendra le risque de ne pas avouer - de ne pas jouer Coulée + car, s'il n'avoue pas, s'il ne joue pas Coulée+ - il est de l'intérêt de l'autre de le faire.

\section{STRATÉGIE ÉVOLUTIONNAIREMENT STABLE}

Les différents cas de figures étant posés, il s'agit d'estimer leur évolution à travers le temps. Pour cela, faisons une intrusion dans la Théorie des jeux évolutionnaire et son concept de "Stratégie Évolutionnairement Stable" (SES) : une combinaison de tactiques qui, si elle est adoptée par les membres d'une communauté, ne peut être améliorée par aucune autre stratégie.

J'ai l'intuition, écrit sans nuance Dawkins [1989], que nous pourrons en
venir à considérer l'invention du concept de SES (Stratégie
Évolutionnairement Stable) comme l'un des progrès les plus importants en
matière de théorie de l'évolution depuis Darwin [p. 122].

On doit ce concept à Maynard Smith [1982]. Il est le premier à s'être servi de la Théorie des jeux à des fins éthologiques - études scientifiques des mœurs et des comportements en situation. Développée dans Evolution and the Theory of games, son hypothèse de « Evolutionary Stable Strategy » fut notamment inspirée par l'observation des animaux sociaux [Hamilton, 1972].

Prenons un exemple pour illustrer la SES. Supposons qu'il y ait deux tactiques possibles : jouer Léopard de mer - désigné à la suite : phoque ; et jouer Lion de mer désigné : otarie (Maynard Smith illustre la SES par d'autres animaux : Faucon contre Colombe ; mais ces derniers sont moins adaptés à notre réflexion sur les tactiques de nage). Les léopards de mer sont les représentants de la famille des Phocidaes (phoques); les lions de mer, les représentants des otariidés (otaries). En milieu aquatique, les otaries utilisent leurs membres antérieurs pour se propulser, tandis que les phoques se meuvent en ondulant de tout leur corps - dans une coordination plus adaptée à la vitesse [Fish, 1996].

Imaginons un cas extrême mais fréquent dans la nature : celui de la survie. Les proies viennent à manquer. Plus rapide, la technique de nage des phoques est coûteuse en énergie. Lorsqu'ils se disputent une chasse, les phoques rentrant bredouilles sont épuisés, incapables de repartir. Si par contre phoque est en compétition avec otarie, cette dernière ne cherchera pas à tenir la cadence et ne demandera pas son reste. Enfin, si les otaries se disputent une chasse, leur manque de vitesse pure fera perdre du temps mais peu d'énergie. 
Nous allouons arbitrairement des «points» aux otaries et aux phoques en fonction de la situation. Le vainqueur de la chasse empoche +50 . Le vaincu a 0 . Probablement mortelle en période de disette, une course folle sans prise coûte -100. Et on donne -10 pour une simple perte de temps.

L'essentiel n'est pas de savoir qui triomphera d'une chasse à courre opposant phoque (léopard de mer) à otarie (lion de mer); tout le monde connaît la réponse. Nous voulons savoir si Phoque ou Otarie représente une SES. Autrement dit, après plusieurs saisons carencées en nourriture, quelle proportion de phoques et de otaries restera? (Répétons que otarie et phoque sont des tactiques).

Imaginons une colonie de pinnipèdes exclusivement composée d'otaries. Les courses inter otaries rapportent au vainqueur non pas +50 , mais $+50-10=+40$, compte tenu du temps perdu pour la chasse. Le perdant obtient : $0-10=-10$. En moyenne, l'espérance de gain des otaries jouant entre elles est donc de +15 (en supposant une probabilité de prise égale à $1 / 2$ ).

Supposons à présent que quelques phoques s'immiscent au sein de ce pool dominé par les otaries. Sûrs de pouvoir les prendre de vitesse à la chasse, les rares phoques possèdent une espérance moyenne de gain maximale de +50 . C'est mieux que le +15 des otaries. Ils finissent par grossir les rangs et s'imposer.

Les envahisseurs occupent la place. Âprement disputées, les courses inter phoques rapportent : +50 au vainqueur et -100 au vaincu (pour des raisons que j'ai évoquées plus haut). Supposons que phoque puisse espérer remporter une course sur deux. Dans une fourchette de scores s'étendant de -100 à +50 , cela lui assure la piètre somme de -25 . C'est son espérance de gain conditionnel s'il joue contre d'autres membres de sa famille.

Imaginons maintenant qu'au fil des saisons quelques otaries survivent au milieu de cette horde de phoques. Certes, elles éviteront de concurrencer les léopards de mer à la chasse : elles ne sont pas aussi bien dotées pour la vitesse. Leur score de 0 , sera toutefois plus enviable que celui des phoques. Si 0 représente la frontière incertaine entre la vie et la mort, les quelques otaries ont de meilleures chances de survie que les nombreux phoques (-25). Elles vont se multiplier. Et nous revenons en boucle au début de l'histoire, quand l'espace était occupé par une colonie de lions de mer.

Avec le système de points arbitraires retenus, la SES s'obtient en jouant 7 fois sur $12(7 / 12)$ avec phoque et 5 fois sur $12(5 / 12)$ avec otarie (selon le mode de calcul utilisé en section 2, CAS 4 et 5). Ce qui est remarquable ici, c'est que deux populations concurrentes survivent en autorégulant leurs effectifs. Si les léopards de mer excèdent les $7 / 12^{\mathrm{e}}$ de la population totale (formée des deux familles), ces derniers finissent par s'autodétruire et les lions de mer reprennent le dessus jusqu'à atteindre et dépasser les $5 / 12^{\mathrm{e}}$ de l'effectif total. Alors ils deviennent à leur tour trop nombreux et ainsi de suite, jusqu'à l'équilibre (SES).

Appliquons à présent la SES aux 6 cas confrontant Coulée + à Surface+ (de façon anecdotique on aura remarqué que Coulée+ s'apparente plutôt à phoque et Surface + à otarie). Le CAS 3 de la Section 2 est sans équivoque. Même si quelques trublions de la natation s'immiscent au sein du pool des nages instituées, ce dernier plus solide résistera à l'envahisseur. Surface+ est la SES. Il est curieux d'observer que cette possibilité - sur 6 possibles - soit la seule attestée par la fédération de natation.

Pour les cas suivants (en commençant par 1 et 2), soit $p(t)$ la fraction accueillant les Surface+. On peut simplifier $p(t)$ à $p$ dans ce qui suit, mais il ne faut pas oublier que $p$ dépend du temps. Si $p=1$, c'est-à-dire si tous les nageurs se précipitent en surface, il 
n'y a aucune raison que cela change (équilibre de Nash). Mais l'inverse est également vrai : si tous les nageurs avaient singé les mammifères marins avant d'imiter les bateaux, on n'aurait jamais inventé le Papillon, le Dos crawlé, la Brasse ou le Crawl ((Coulée+, Coulée+) est aussi un équilibre de Nash en CAS 1 et 2). Et, dans La parabole des chasseurs, il suffit que le pool de techniques instituées (Surface+) en vienne à comprendre un ou plusieurs intrus $(0<p<1)$ pour que les stratégies convergent, de compétitions en compétitions, vers ce second équilibre : $p=0$, la SES des CAS 1 et 2 (Figure 2). Pour le calcul de l'équation aboutissant aux courbes des Figures 2 et suivantes, on pourra se reporter au chapitre 9 du livre de Ken Binmore [1992] : Fun and Games.

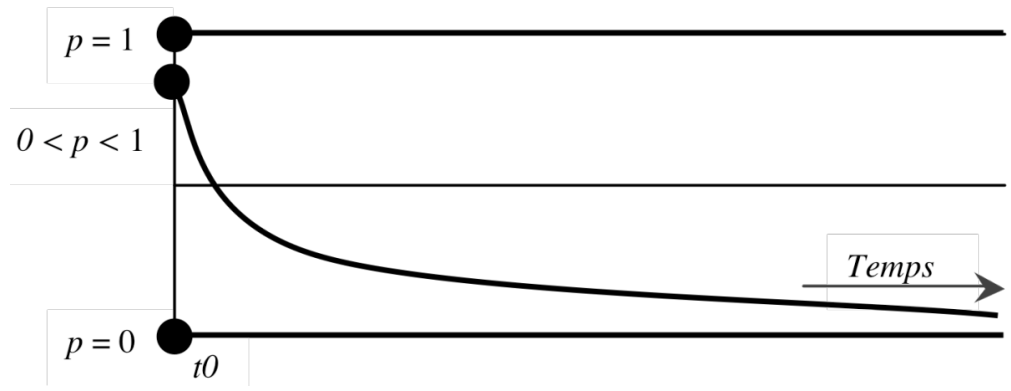

FIGURE 2. Évolution des nages dans les CAS 1 et 2 (La parabole des chasseurs) Si aucun intrus (Coulée+) ne se manifeste au fil des compétitions, la population des Surface+ demeure inchangée $(p=1)$. Mais il suffit qu'un seul émerge pour que l'effectif des Surface+ s'amenuise et tende inéluctablement vers zéro $(p=0)$. Si la population de Coulée+ existait avant l'arrivée de Surface+, ces derniers n'auraient pas vu le jour (adapté de Binmore [1992])

Résumés par Poule mouillée, les CAS 4 et 5 cultivent l'équivoque. Aucun des points de repos $p=1$ ou $p=0$ n'est stable. Le premier correspond toujours à une population dans laquelle seul le réplicateur Surface+ est présent. Un réplicateur est tout ce dont on fait des copies [Dawkins, 1989]. Les techniques de surface se copient avec fidélité et fécondité dans les centres d'entraînement et les bassins de compétition. S'il devait y avoir l'intrusion d'une fraction même minuscule de Coulée + dans le pool de nages, alors cette fraction augmenterait au fil du temps jusqu'à ce que la proportion de réplicateurs Surface+ et Coulée+ soit égale $(p=1 / 2$, Figure 3). Cette situation correspond à la SES. Remarquons que dans l'exemple de phoque vs otarie, les fonctions de $p$ aboutissant à la SES sont du même type qu'en Figure 3, mais avec un attracteur local égal à $5 / 12$ au lieu de $1 / 2$.

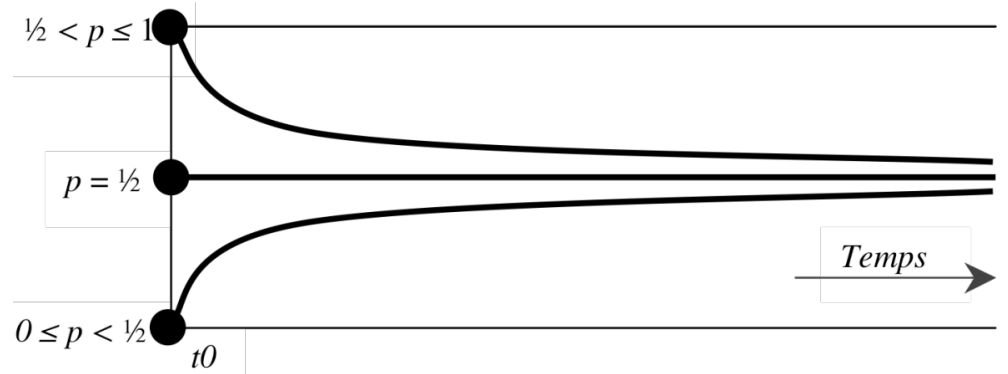

FIGURE 3. Évolution des nages dans les CAS 4 et 5 (Poule mouillée) Aucun point de repos $p=0$ ou $p=1$ n'est stable. Au fur et à mesure des rencontres, la part de chaque population s'équilibre : il y aura autant de Surface + que de Coulée+ $(p=1 / 2)$. (Adapté de Binmore [1992]) 
Le dernier cas, dénommé Dilemme du prisonnier (CAS 6), donne l'avantage aux perturbateurs sous-marins. Puisque l'unique équilibre de Nash du jeu est (Coulée+, Coulée +), il n'est pas étonnant que le seul attracteur local soit $p=0$. Ce qui correspond au cas où les nageurs prennent Berkoff pour modèle (Figure 4). Le point de repos $p=0$ correspond à la SES.

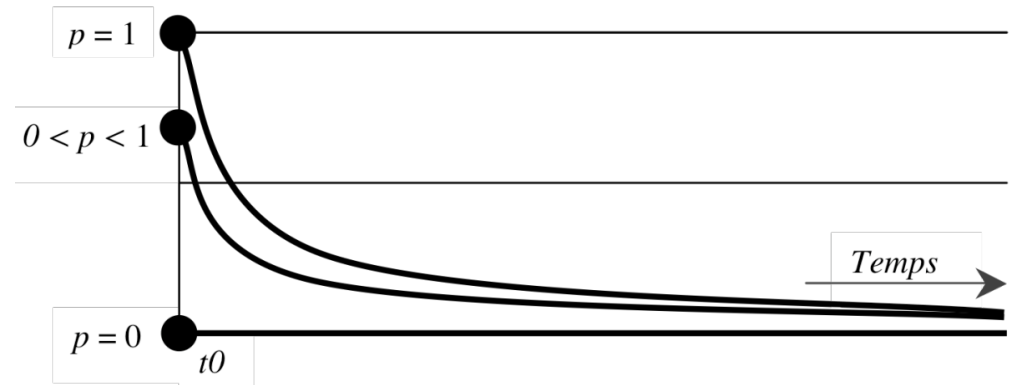

FIGURE 4. Évolution des nages dans le CAS 6 (Dilemme du prisonnier)

Puisque le seul équilibre de Nash : (Coulée+, Coulée+) devient le seul attracteur local $(p=0)$, la cinquième nage prend le dessus sur les nages de surface (adapté de Binmore [1992])

Si l'on accorde une équiprobabilité aux 6 cas de figures et compte tenu de l'apparition effective de nouveaux savoirs nager en immersion, la probabilité $s$ que le réplicateur en place $($ Surface +$)$ garde la vedette est : $s=(p(t)$ CAS1 $+p(t)$ CAS2 + $p(t) \mathrm{CAS} 3+p(t) \mathrm{CAS} 4+p(t) \operatorname{CAS} 5+p(t) \operatorname{CAS} 6) / 6=(0+0+1+1 / 2+1 / 2+0) / 6=1 / 3$. Il y a donc deux chances sur trois pour que «la cinquième nage » (dauphin) s'impose comme nouvelle technique du corps.

\section{VERS L'ÉMERGENCE D'UNE CINQUIÈME NAGE}

Les nageurs recourant outrageusement aux coulées sont les véhicules discrets des nouveaux réplicateurs de la natation sportive. La nage en immersion est amenée à prendre le dessus sur les quatre autres. Cela bien sûr, si l'évolution des façons de nager se plie aux exigences de la rationalité Nashienne, purement égoïste et opportuniste. Pour autant, en même temps que nous parions sur l'émergence d'une cinquième nage récemment illustrée au plus haut niveau ${ }^{2}$, nous reconnaissons le caractère arbitraire du savoir-nager. À l'instar de toutes les techniques du corps, les techniques de nage sont artificielles [Mauss, 1934]. Elles ne sauraient se soumettre aux pressions de l'Horloger aveugle (Dawkins [1986] utilise cette expression pour désigner l'aveuglement de la sélection naturelle). Jean Piaget [1932] - par la psychogénétique, John Harsanyi [1977] - par la formalisation mathématique, et Robert Axelrod [1997] - par la Théorie des jeux expérimentale - ont mis en évidence l'existence d'une seconde forme de rationalité guidée par la morale et non plus par les intérêts singuliers. C'est à ce titre que les instances officielles légifèrent : au nom de l'intérêt collectif, pour maximiser le niveau de satisfaction moyen de tous les joueurs.

L'institution sanctionne avec autorité les resquilleurs, les fraudeurs, les passagers clandestins. On contrôle le paiement des impôts, on limite les quotas de pêche, on évite de gâcher, de polluer et... on disqualifie les nageurs s'immergeant au-delà des quinze mètres. Pour le bien collectif. On comprend qu'il faille redistribuer les richesses, éviter

\footnotetext{
${ }^{2}$ www.floswimming.org/videos/speaker/1975-taylor-hill.
} 
de dégrader la planète pour les générations à venir, etc. Mais dans le cas de la natation, de quel intérêt général s'agit-il ? Défendre la pérennité des quatre techniques instituées ou assurer les meilleures prestations aquatiques ? S'il s'agit juste de garantir l'égalité des chances au départ, pourquoi ne pas légaliser une cinquième nage pour préserver la spécificité des quatre autres ? Pour l'heure, et sous couvert de savantes réglementations, la Fédération entretient artificiellement la validité du CONSTAT 1 en dressant les nageurs à rester en surface.

Les conclusions issues de la Théorie des jeux invitent à la mutinerie. En sport, les joueurs ne gagnent pas en faisant bien mais en faisant mieux : l'intérêt des nageurs rationnels est de chasser le lièvre, de ne pas toujours être le premier à sauter de la voiture en marche ou encore d'avouer les crimes de leur coéquipier !

\section{BIBLIOGRAPHIE}

AXELROD R. [1997], The complexity of cooperation: Agent-based models of competition and collaboration, Princeton, Princeton Studies in Complexity.

BINMORE K. [1992], Fun and Games: a text on Game theory, Lexington (Mass.), D.C. Heath \& Company.

BOUDON R. [1977], Effet pervers et ordre social, Paris, Presses Universitaires de France.

CATTEAU R. [2008], La natation de demain, Biarritz, Atlantica.

COLLARD L. [2007], "Average swimming speeds for 6 sections of the 50-meter backstroke race". Intern. J. of Perf. Anal. in Sport, Vol. 7, n 3, p. 37-45.

COLLARD L. [2009], La cinquième nage. Natation et théorie de l'évolution, Biarritz, Atlantica.

COUNSILMAN J. E. [1968], The science of swimming, Englewood Cliffs (NJ), Prentice-Hall.

DAWKINS R. [1989], The Selfish Gene, Oxford, Oxford University Press.

DAWKINS R. [1986], The Blind Watchmaker, New York, W. W. Norton \& Company, Inc.

FISH F.E. [1993], "Power output and propulsive efficiency of swimming bottlenose dolphins", J. Exp. Biol. 185, p. 179-193.

FISH F.E. [1996], "Transitions from drag-based to lift-based propulsion in mammalian swimming", Amer. Zool. 36, p. 628-641.

HAMILTON, W.D. [1972], "Altruism and related phenomena, mainly in social insects", Annual Review of Ecology and Systematics 3, p. 193-232.

HARSANYI J. [1977], “Morality and the theory of rational behavior”, Social Research 44, p. 623-656.

HERTEL H. [1966], Structure, form, movement, New York, Reinhold.

HOLT L.E. [1989], Swimming velocity with and without lift forces, Sports Sciences Laboratory, Canada, Dalhousie University [unpublished paper].

MAGLISCHO E.W. [2003], Swimming fastest, Human Kinetics, Champaign (Il), Leeds.

MAUSS M. [1934], «Les techniques du corps », Journal de Psychologie, XXXII, 3 \& 4, p. 366-383.

MAYNARD SMITH J. [1958], The Theory of evolution, London, Penguin Books.

MAYNARD SMITH J. [1982], Evolution and the Theory of games, Cambridge, Cambridge University Press.

NASH J. [1950], "Equilibrium points in n-person games", Proceedings of the National Academy of Sciences of the USA 36, p. 48-49.

NASH J. [1951], “Non-cooperative games”, Annals of Mathematics 54, p. 286-295.

PARLEBAS P. [1986], Éléments de sociologie du sport, Paris, Presses Universitaires de France. 
PARLEBAS P. [2005], "Mathématiques, jeux sportifs, sociologie", Mathématiques et Sciences humaines 170 , p. 5-9.

PIAGET J. [1932], Le jugement moral chez l'enfant, Paris, Presses Universitaires de France.

ROUSSEAU J.-J. [1964], « Du contrat social. Écrits politiques» [1756], CEuvres complètes III, Paris, Gallimard, La Pléiade.

SANDERS R.H. [1997], "Extending the 'Schleihauf' model for estimating forces produced by a swimmer's hand”, B.O. Eriksson \& L. Gullstrand (eds), Proceedings of the XII FINA World Congress on Sports Medicine, p. 421-428, Göteborg (Sweden), Chalmers Reproservice.

SCHLEIHAUF R.E. [1978], Swimming propulsion: a hydrodynamic analysis, Fort Laudedale (Florida), American Swimming Coaches Association.

SPRIGINGS E.J., KOEHLER J.A. [1990], “The choice between Bernoulli's or Newton's model in predicting dynamic lift", International Journal of Sport Biomechanics 6, p. 235-245.

SPOOR F., BAJPAI S., HUSSAIN S.T., KUMAR S.T., THEWISSEN J.G. [2002], "Vestibular evidence for the evolution of aquatic behaviour in early cetaceans", Nature 417, p. 163-166.

THEWISSEN J.G., HUSSAIN S.T., ARIF M. [1994], "Fossil evidence for the origin of aquatic locomotion in archaeocete whales", Science 263, p. 210-212.

THEWISSEN J.G., COOPER L.N., CLEMENTZ M.T., BAJPAI S., TIWARI B.N. [2007], "Whales originated from aquatic artiodactyls in the Eocene epoch of India”, Nature 450 (7173), p. 1190.

TUCKER A. [1950], A two person dilemma, Mimeo, Standford University.

VAN EERSEL P. [1993], Le cinquième rêve, Paris, Le livre de poche. 